\title{
Production of Thick Crude through Concentric Coiled Tubing
}

Mounir Ababou (Technical Manager, Baker Hughes, Oman)

The drilling of exploration wells in thick Oil reservoirs has proven to be a major challenge for Oman Operator when it came to establish the presence of Oil in these wells. Traditional method consisted of using Nitrogen lifting to recover the oil, but this has proven to be very uneconomical option due to the extremely low pressure reservoirs. The option to recover the fluids using Beam pumps out of these low pressure/ high viscosity wells is possible, but as these wells are under exploration program, the client needs to assess the presence of Oil first before committing to bring in Beam pump/ PCP.

A new method to try to establish the presence of Oil out of these wells consisted of using a Concentric Coiled tubing, which allows to produce wells with very low bottom hole pressure. At the end of the Concentric Coil is a special Vacuum tool. This technology is widely used in Oman to perform sand clean outs, logging and Acid stimulation, however, the new method for producing thick crude consisted of circulating hot water down the concentric coiled tubing, so that it would act as a "radiator" to heat up the bottom hole area, then engage the vacuum tool to produce thick crude to surface. 